\title{
Design of WDM Cross Connect Based on Interleaved AWG (IAWG) and a Phase Shifter Array
}

\author{
Yueting Wan and Rongqing Hui
}

\begin{abstract}
A general design rule for interleaved arrayed waveguide gratings (IAWGs) is derived. A $1 \times N$ WDM switch that is based on a phase shifter array, which is sandwiched between two $N$-IAWGs, is systematically analyzed. A practical design example of a $1 \times 4$ nonblocking wavelength division multiplexing (WDM) switch is detailed to illustrate the design steps and the required phase assignment of each phase shifter for various output states. Based on the reciprocity principle, a simplified structure of the $1 \times N$ WDM switch is proposed, which requires only one $N$-IAWG with total reflection implemented at the end of each phase shifter. This type of $1 \times N$ WDM switch can be used as a fundamental building block to construct the nonblocking $N \times N$ WDM all-optical switch that will be indispensable for future all-optical networks.
\end{abstract}

Index Terms-Arrayed waveguide grating (AWG), optical cross connect (OXC), optical switch, photonic device, planar lightwave circuits (PLCs), wavelength division multiplexing (WDM).

\section{INTRODUCTION}

$\mathbf{T}$ HE WAVELENGTH division multiplexing (WDM) technique has been widely applied in fiber-optic networks by effectively utilizing the wide wavelength windows of optical fibers and by providing an additional degree of freedom in optical networking [1]. In the development of future WDM optical networks, it is critical to have optical switches that can optically route wavelength channels from any input port to any output port and, thus, fully realize the functionality of nonblocking $N \times N$ optical switch.

Arrayed waveguide gratings (AWGs) have been important devices which are generally used in WDM optical systems as wavelength multiplexers and demultiplexers. Refractive index tuning of arrayed waveguides between the two star couplers in an AWG has been introduced to adjust and optimize the transfer function of the device. This index tuning in AWG can also be used to perform the WDM switch with proper device structure designs [2]-[4]. In silica-based AWGs, index tuning can be accomplished by locally heating each individual waveguide branch. However, this thermal-based tuning is too slow to satisfy the requirement for optical packet switching. In order to overcome the speed limit of thermal tuning, semiconductor materials such as GaAs, InP or, recently, GaN, have also been used to make AWG devices, in which refractive indexes of

Manuscript received August 14, 2006; revised February 14, 2007. This work was supported by the National Science Foundation under Grants ECS-0123450 and ECS-0532610.

Y. Wan was with the Department of Electrical Engineering and Computer Science, University of Kansas, Lawrence, KS 66044 USA. He is now with Harbor Branch Oceanographic Institution, Fort Pierce, FL 34946 USA.

R. Hui is with the Department of Electrical Engineering and Computer Science, University of Kansas, Lawrence, KS 66044 USA.

Digital Object Identifier 10.1109/JLT.2007.896808 arrayed waveguides can be adjusted by carrier injection. In general, the speed of a carrier-induced index change can be in the subnanosecond level, which is many orders of magnitude faster than thermal tuning, and therefore, it can potentially be used to support all-optical packet switches in the future.

A number of different designs and device structures have been proposed to realize AWG-based optical switches [2], [3], [5], which utilize a unique interleaved-AWG (IAWG) structure. A $2 \times 2$ nonblocking wavelength switch has been demonstrated using this approach, and it can potentially be extended to realize the switch size of $N \times N$ [2]. However, the $N \times N$ switch illustrated in [2] is not truly "any to any." Instead, its switching functionality is cyclic [5]. Therefore, truly any-toany nonblocking optical switch functionality cannot be realized directly using the structure proposed in [5]. In addition, a detailed general design rule of IAWGs is thus far unavailable. Although, from a switch architecture point of view, a truly anyto-any $N \times N$ nonblocking switch can be constructed by a group of $1 \times N$ switches [6], the design of the $1 \times N$ switch for a single wavelength, using an IAWG-based planar lightwave circuit (PLC), has not been fully investigated, and therefore, its applications to construct a truly any-to-any $N \times N$ wavelength switch could not be fully explored.

In this paper, we propose a fully functional nonblocking $N \times N$ WDM switch architecture that uses a group of IAWGbased $1 \times N$ optical switches as building blocks. We then present a detailed description of a self-consistent design rule of a $1 \times N$ all-optical switch, which is a basic component to realize a truly any-to-any $N \times N$ WDM all-optical switch. Numerical examples are then presented to illustrate the device characteristics. Specifically, a design example of a $1 \times 4$ nonblocking WDM switch is presented, and the phase assignment at each phase shifter is provided for various routing states. Finally, we propose a simplified structure of a $1 \times N$ WDM switch based on a single IAWG with a total reflection at the end of each phase shifter. Design considerations and device characteristics will be discussed. It is also important to note that the proposed $1 \times N$ switch structure can be constructed on a PLC with no waveguides crossing; therefore, it can be monolithically integrated with other devices to create sophisticated optical circuits with various functionalities.

\section{ApproACH OF $N \times N$ All-Optical SWitch}

It is well known that any-to-any nonblocking wavelength switches are indispensable for optical networks. However, the design of a true any-to-any optical switch using PLC technology is not an easy task because of its complexity and 


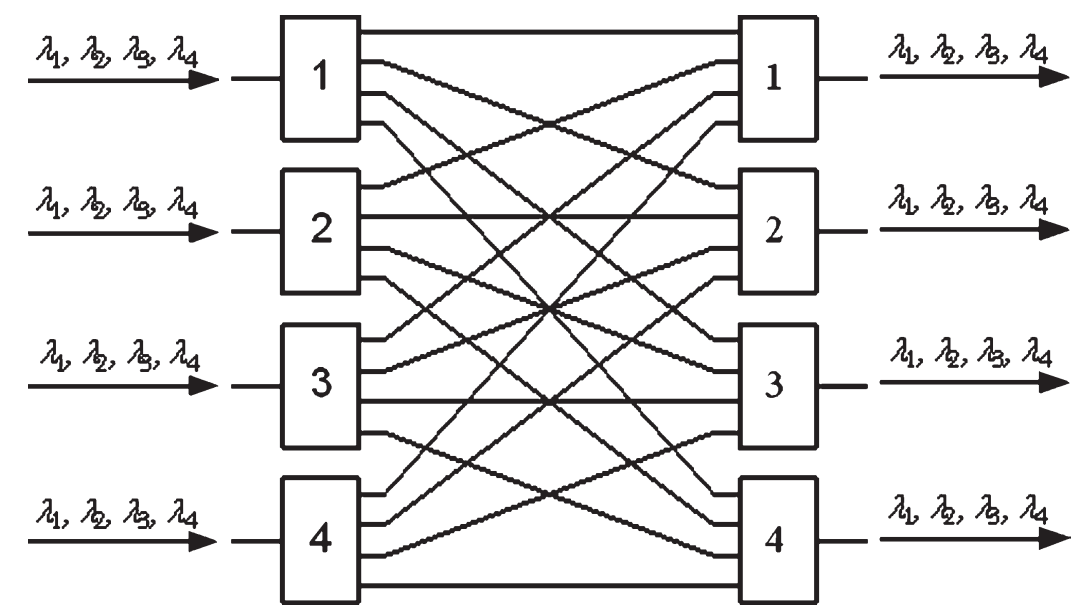

Fig. 1. Scheme of a $4 \times 4$ all-optical switch.

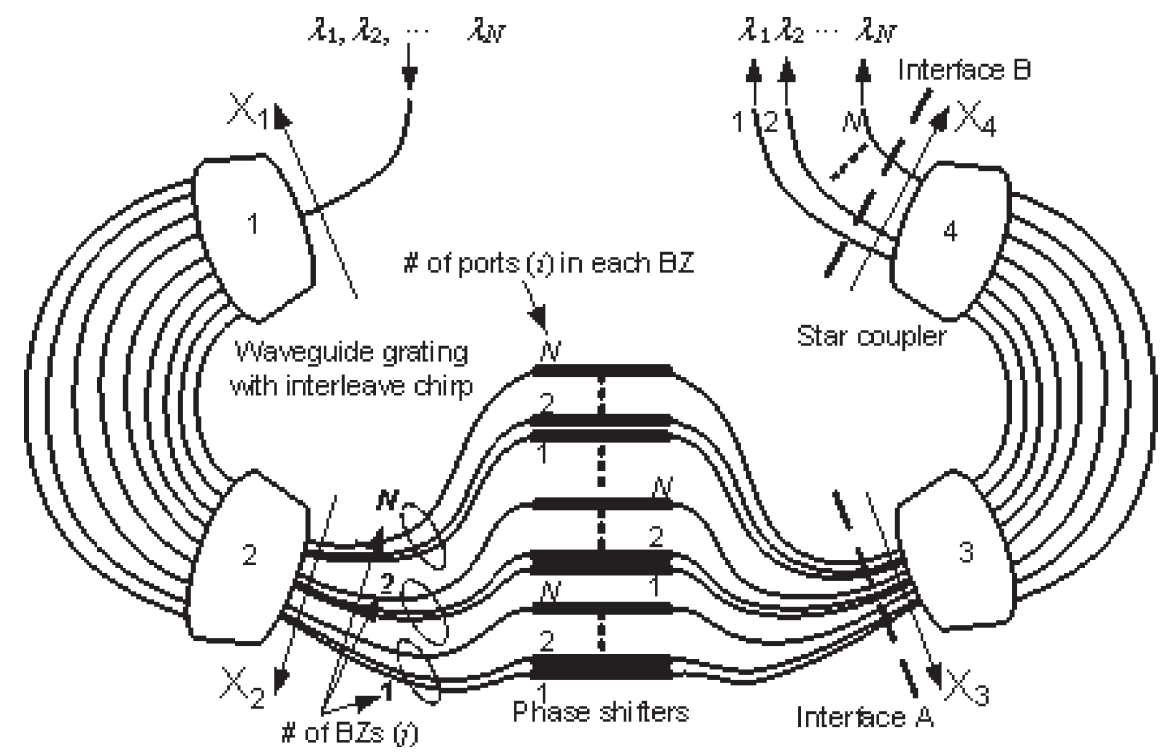

Fig. 2. Schematic diagram of a $1 \times N$ all-optical switch.

because it has not been demonstrated thus far. One modular approach to realize the $N \times N$ switch functionality is to combine a group of $1 \times N$ optical switches. To illustrate this architecture, Fig. 1 shows a $4 \times 4$ nonblocking wavelength switch, which consists of eight $1 \times 4$ wavelength switches with four of them at the input side and the other four at the output side. For each $1 \times 4$ optical switch at the input side, it can route any of the four wavelength channels $\lambda_{1}$, $\lambda_{2}, \lambda_{3}$, and $\lambda_{4}$ into any of its four output ports. Similarly, each output $4 \times 1$ optical switch combines the four received wavelength channels and feeds them into one output fiber. Since the functionality of the optical switch is determined by the four input switches, the four output switches are performing a redundant operation and can simply be replaced by four $4 \times 1$ optical star couplers. However, with this simplified option, there will be a $10 \log (4)=6 \mathrm{~dB}$ intrinsic combining loss for the optical signal because the power combiner is not wavelength selective.

Since $1 \times N$ wavelength switches are fundamental building blocks to realize an $N \times N$ nonblocking wavelength switch, our focus will be on the development of a general design rule for a $1 \times N$ wavelength switch in the following sections.

\section{BRIEF DESCRIPTION OF A $1 \times N$ ALL-OptICAL SWITCH}

A schematic design of a $1 \times N$ optical switch is shown in Fig. 2. Similar to the study in [5], this optical switch consists of two IAWGs and an array of phase shifters between them. However, instead of having two or more input ports at the input side, as suggested by Doerr [5], the structure shown in Fig. 2 has only one input port. In this figure, star couplers 1 and 2 form the input $N$-IAWG, and star couplers 3 and 4 form the output $N$-IAWG. Between these two $N$-IAWGs, there are $N^{2}$ waveguide phase shifters that are divided into $N$ equalnumbered subsets. The input $N$-IAWG is designed in such a way that the power of the input signal at wavelength $\lambda_{i}$ is directed into all phase shifters labeled with the index $l_{i j}$, where $i$ is the index of ports within each Brillouin zone (BZ), and $j=1,2, \ldots, N$ is the index of BZs, as shown in Fig. 2. The 


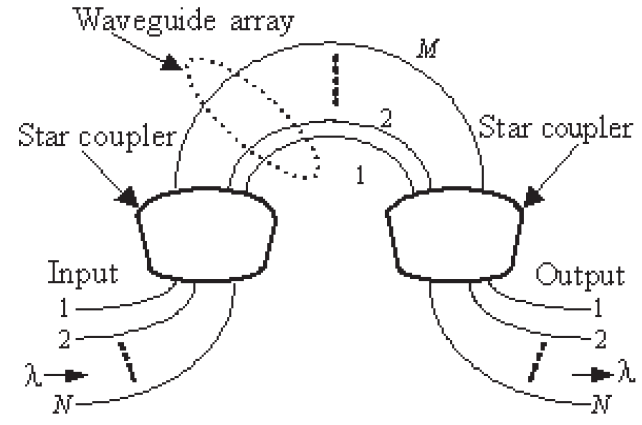

(a)

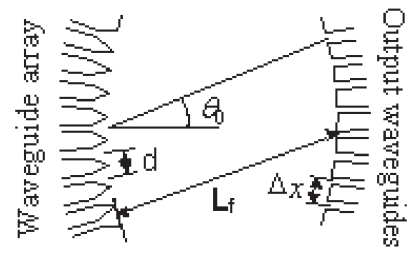

(b)

Fig. 3. (a) AWG structure. (b) Configuration of the star coupler.

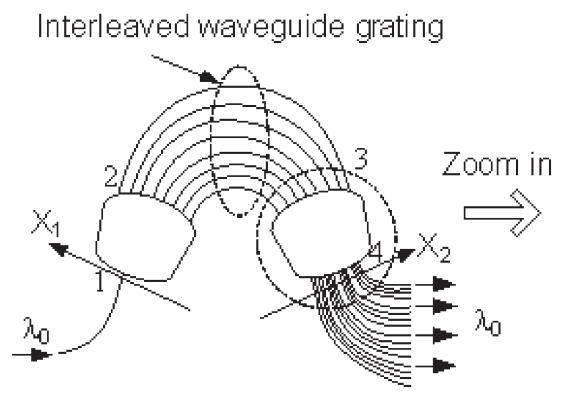

(a)

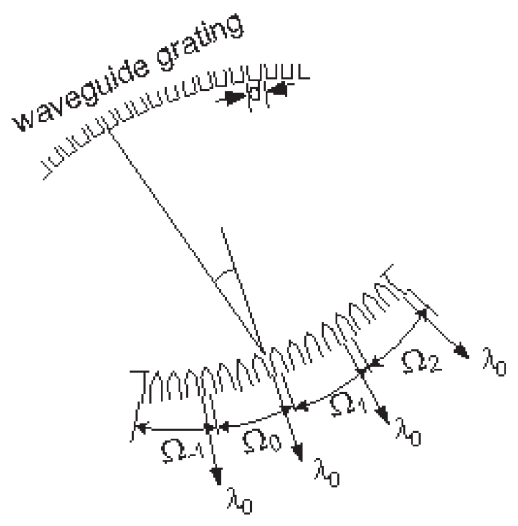

(b)

Fig. 4. Diagram of output port distribution for an $N$-IAWG. (a) $N$-IAWG, where $N=4$. (b) Close-up of the output star coupler, where $\Omega_{i}(i=-1,0,1,2)$ denotes the new BZs of this 4-IAWG. These new BZs were split from the central BZ of a conventional AWG before it was interleaved.

concept of BZ will be explained later. Ideally, there should be an equal amount of signal power of wavelength $\lambda_{i}$ directed into each $l_{i j}$ phase shifter. This allows the destination of the input signal at wavelength $\lambda_{i}$ to be totally controlled by all $l_{i j}$ phase shifters, and therefore, the $1 \times N$ switching functionality can be realized. The switching functionality will be further explained in the next section.

\section{DESIGN OF THE $N$-IAWG}

\section{A. Functionality of N-IAWG}

In Section III, we mentioned that the $N$-IAWG is designed in such a way that the input signal with wavelength $\lambda_{i}$ is distributed into all the output waveguides that are connected with the phase shifters labeled with $l_{i j}$. (To be convenient, we will also use $l_{i j}$ to denote these output waveguides directly.) Moreover, for optimum operation, the amount of signal power entering each waveguide in this group should be the same.

The above condition was mentioned for the $N$-IAWG that has only one input port, as shown in Fig. 2. It can be extended to a general $N$-IAWG which has $N$ input ports. Star couplers 4 and 3 in Fig. 2 form such a general $N$-IAWG if star coupler 4 is used as the input and star coupler 3 is used as the output. Under this assumption, when the separation between the waveguide ports at interface $\mathrm{B}$ is arranged properly, the $N$-IAWG can direct the signal of wavelength $\lambda_{i}$ into the same waveguide ports $l_{i j}$ at interface $\mathrm{A}$, no matter which waveguide port at interface B is chosen as an input port. The amount of the power coupled into each of the waveguide ports $l_{i j}$ at interface $\mathrm{A}$ should also be the same.

Based on the above description, the output power is always evenly distributed among the waveguides labeled with $l_{i j}$ at interface $\mathrm{A}$ for a signal at wavelength $\lambda_{i}$, no matter which waveguide port at interface B it originally enters. However, the phase distribution of the output signal is different when a different waveguide port at interface B is used as an input port. Based on reciprocity, if a signal with wavelength $\lambda_{i}$ is uniformly launched into all waveguides labeled $l_{i j}$ at interface $\mathrm{A}$, we should be able to direct the signal into any one of the waveguide ports at interface B by adjusting the phase of each input properly. This explains the switching functionality of the structure in Fig. 2. More details about this switching functionality will be explained later.

\section{B. From AWG to IAWG}

This section is dedicated to show how to construct an $N$ IAWG based on a conventional AWG. For convenience, we use a 4-IAWG as an example. Figs. 3 and 4 show the detailed structures of a conventional AWG and a 4-IAWG, respectively. Similar to an AWG, the IAWG has an input star coupler and an output star coupler. However, the path length difference between adjacent arrayed waveguides for an IAWG differs from 
TABLE I

INITIAL VECTOR INFORMATION FOR CONSTRUCTING A 4-IAWG

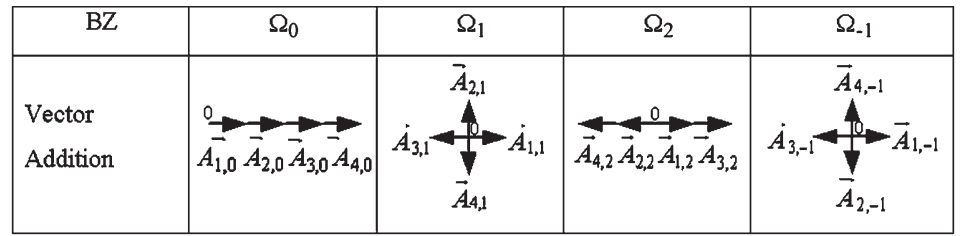

that of an AWG to create a different wavelength-dependent focusing effect. To obtain the appropriate path length differences in the IAWG, we can start with the central phase matching equation of a conventional AWG [7], [8]

$$
n_{s} d \sin \left(\theta_{i}\right)+n_{c} \cdot \Delta L+n_{s} d \sin \left(\theta_{0}\right)=m \lambda
$$

where $m$ is the grating order, $n_{c}$ is the effective refractive index of the arrayed waveguides, $n_{s}$ is the effective refractive index of the slabs of the star couplers, $\Delta L$ is the path length difference between two adjacent waveguides in the array, $d$ is the separation of adjacent waveguides at the star coupler, $\theta_{i}$ is the angle of $i$ th input port with respect to the direction of the central input port, $\theta_{0}$ is the diffraction angle in the output star coupler, and $\lambda$ is the output wavelength. According to the study in [8]

$$
\Delta L=\frac{m \lambda_{0}}{n_{c}}
$$

where $\lambda_{0}$ is the center operating wavelength of the device. Now, we will show how to find the additional waveguide lengths in addition to $\Delta L$ in (2) to construct an $N$-IAWG.

Equation (1) indicates that there is an angular period in the radiation pattern of the output star coupler of an AWG when the diffraction angle $\theta_{0}$ is relatively small. This angular period is defined as a BZ, which is a very useful concept in the design of an IAWG. From (1), the angular width $\Delta \theta$ of a BZ can be found as [9]

$$
\Delta \theta=\frac{\lambda}{n_{s} d}
$$

The angular separation between $-\Delta \theta / 2$ and $\Delta \theta / 2$ is commonly referred to as the central BZ. Only the output in the central BZ is generally considered for an AWG, since practically, most of the signal energy is concentrated in this area.

Suppose there are $M$ arrayed waveguides between the two star couplers, they can be divided into four subsets with the $i$ th subset composed of waveguides $i, i+4, i+8, i+12, \ldots$, where $i=1,2,3,4$. In such an arrangement, if any three of the four subsets are removed, the remaining subset is still an AWG, but the path length difference between the adjacent waveguides will be $4 \Delta L$, and the waveguide separation at the input and the output star couplers will be $4 d$. If a single wavelength $\left(\lambda_{0}\right)$ signal is coupled into this remaining subset, the central phase match condition of this reduced AWG can be obtained from (1) as

$$
n_{s}(4 d) \sin \left(\theta_{i}\right)+n_{c} \cdot(4 \Delta L)+n_{s}(4 d) \sin \left(\theta_{0}\right)=m^{\prime} \lambda_{0}
$$

where $m^{\prime}$ is the grating order of this reduced AWG. The angular separation between two adjacent BZs at the output of the star coupler of this reduced AWG is

$$
\Delta \theta^{\prime}=\frac{\lambda_{0}}{n_{s}(4 d)} .
$$

A comparison of (5) and (3) shows that the BZ of the reduced AWG has an angular width which is one fourth that of the original AWG. In other words, the central BZ of the original AWG splits into four new BZs for each of the four subset AWGs. We denote these four new BZs as $\Omega_{-1}, \Omega_{0}, \Omega_{1}$, and $\Omega_{2}$, as shown in Fig. 4(b). Since the four subset arrayed waveguides combine only to form the original AWG, when all the four subsets exist, there is only one maximum in the output field pattern over the entire combined angular region of $\Omega_{-1}, \Omega_{0}$, $\Omega_{1}$, and $\Omega_{2}$, which is the central BZ of the original AWG. This maximum output is due to the interference among the signals from the different subsets. It is convenient to arrange this maximum output port in $\Omega_{0}$. In this case, we see that the signals of wavelength $\lambda_{0}$ from the four subsets of the arrayed waveguides add constructively at its related output port in $\Omega_{0}$ and destructively at the related output ports in $\Omega_{-1}, \Omega_{1}$, and $\Omega_{2}$.

The above explanation can be illustrated in vector expressions. For subset $p$ of the arrayed waveguides, its output optical field at the related output port in $\mathrm{BZ} \Omega_{q}$ can be denoted as vector $\vec{A}_{p, q}$, where in our example, $p=1,2,3,4$ and $q=-1,0,1,2$. We have

$$
\sum_{p=1}^{4} \vec{A}_{p, q}=\left\{\begin{array}{ll}
0, & q=-1,1,2 \\
4 \cdot \vec{A}_{1,0}, & q=0
\end{array} .\right.
$$

The vector additions given by (6) are illustrated in Table I, where we are mainly interested in the relative phase angles between the vectors, and thus, the orientations of vectors $\vec{A}_{1, q}$ are used as the phase references.

Table I shows that although each subset of arrayed waveguides by itself would project the same amount of output power at the related output port in each of these BZs $\left(\Omega_{q}\right)$, the combination of the four subsets creates only one maximum, which is located in BZ $\Omega_{0}$, as the result of vector addition of four field components in each BZ. If the optical field magnitude created by each arrayed waveguide subset is $A_{0}=\left|\vec{A}_{p, q}\right|$, the maximum output optical power in $\Omega_{0}$ will be $16 A_{0}^{2}$.

In an $N$-IAWG, the path length difference between adjacent arrayed waveguides varies so that each original BZ in an AWG, on which the $N$-IAWG is developed, is split into $N$ equally spaced narrower BZs [5]. Thus, a 4-IAWG should have four new BZs $\Omega_{-1}, \Omega_{0}, \Omega_{1}$, and $\Omega_{2}$, as shown in Fig. 4(b). An 
TABLE II

Vector Information for a 4-IAWG With $\pi$ PhASE Shift

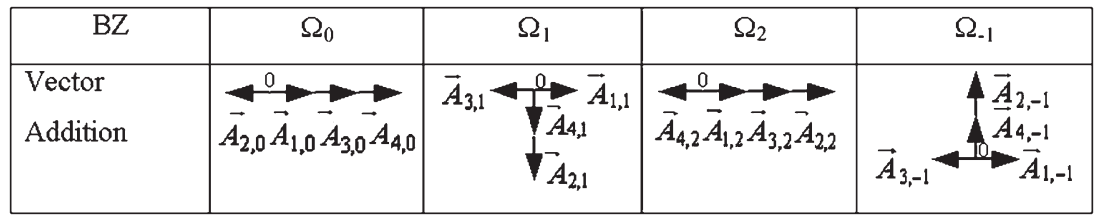

important requirement for the phase array application is that the signal powers from the output ports in the four new BZs should be the same to ensure efficient field interference. That is to say, in this 4-IAWG example, each new BZ should have a quarter of the total output power. In practice, the power transfer function of a star coupler has Gaussian distribution with the maximum in the middle. Most of the optical power will be concentrated in the central region [5]. Therefore, only the four new BZs near the center need to be considered, while the BZs far away from the center can be neglected since the power in these regions is very low.

To obtain the same output power at the output port in each new BZ, we can rotate all the vectors $\vec{A}_{2, q}$ in Table I by $180^{\circ}$. After the rotation, the new phase diagrams of the four vectors are shown in Table II. It demonstrates that this $\pi$ phase rotation makes the optical power be equally split into the four BZs and the output power at each $\mathrm{BZ}$ be $4 A_{0}^{2}$, which is one fourth of the maximum power, as shown in Table I.

Rotating vectors $\vec{A}_{2, q}$ in Table I by $180^{\circ}$ corresponds to changing the phase of the output field emerging from the waveguides of subset 2 by $\pi$. This can be realized by adding an additional length $\lambda_{0 c} / 2$ to all the waveguides in subset 2, where $\lambda_{0 c}=\lambda_{0} / n_{c}$. In general, a 4-IAWG can be realized by adding the additional length $\lambda_{0 c} / 2$ to all the waveguides in any one of the four subsets while keeping the waveguides in three other remaining subsets unchanged.

This design procedure can be further simplified. In the above discussion, all the four BZs were considered in finding the required additional waveguide lengths. Since the initial value of the summation of all the vectors in each of the BZs $\Omega_{-1}$, $\Omega_{1}$, and $\Omega_{2}$ is zero, the magnitudes of the vector summations in these three BZs will always be the same as long as each of vectors $\vec{A}_{2, q}$ rotates by the same amount. This indicates that only one of these three BZs needs to be considered together with BZ $\Omega_{0}$ in the designing of a 4-IAWG. This generalization will be further explained later.

\section{General Design Rule for an N-IAWG}

Based on the above example, a general rule in the design for an $N$-IAWG can be obtained as summarized in the following steps.

1) Divide the $M$ arrayed waveguides in the AWG into $N$ subsets. In each subset, $N \cdot \Delta L$ is the path length difference between adjacent waveguides, and $N \cdot d$ is the arrayed waveguide separation at the input and output star couplers.

2) Find the initial vector information as in Table I. As mentioned above, we only need to consider one more
$\mathrm{BZ}$, for example $\Omega_{1}$, besides the central BZ $\Omega_{0}$, where the maximum initial output power $N^{2} A_{0}^{2}$ exists. Similar to (6), the initial values of the sums of the vectors in BZs $\Omega_{0}$ and $\Omega_{1}$ are

$$
\begin{aligned}
& \vec{V}_{\Omega_{0}}=\sum_{p=1}^{N} \vec{A}_{p, 0}=N \cdot \vec{A}_{1,0} \\
& \vec{V}_{\Omega_{1}}=\sum_{p=1}^{N} \vec{A}_{p, 1}=0 .
\end{aligned}
$$

Based on this, similar phase diagrams, as shown in Table I, can be obtained.

3) Rotate any pair of vectors $\vec{A}_{p, 0}$ and $\vec{A}_{p, 1}$ by the same angle $\delta_{p}$, where $p=2, \ldots N$, and then, rotate as many pairs as necessary until the following condition is met:

$$
\vec{V}_{\Omega_{0}}=\sum_{p=1}^{N} \vec{A}_{p, 0}=\sum_{p=1}^{N} \vec{A}_{p, 1}=\vec{V}_{\Omega_{1}}=\sqrt{N} \cdot \vec{A}_{1,0} .
$$

At this point, one can record all the angular values $\delta_{p}$, and the additional length needed for the waveguides in subset $p$ can be calculated as

$$
\Delta L_{\mathrm{add}}=\frac{\delta_{p}}{2 \pi} \cdot \lambda_{0 c}
$$

where $\delta_{p}$ is in radians. Since $0 \leq \delta_{p}<2 \pi, \Delta L_{\text {add }}$ is always less than $\lambda_{0 c}$. Finding $\Delta L_{\text {add }}$ values is an essential step in the design of an $N$-IAWG.

\section{Comparison With Previous Results}

The general rule discussed above can be used to calculate the additional lengths $\Delta L_{\text {add }}$ needed in arrayed waveguides to transfer an AWG into an $N$-IAWG. Multiple sets of $\Delta L_{\text {add }}$ can be obtained by following the general design rule, for a particular $N$, to achieve the same result. Table III(a) shows examples of $\Delta L_{\text {add }}$ that were calculated by using this general rule for $N=$ $2,3,4,5,6,7$, and 8 . As $N$ increases, the number of solutions of $\Delta L_{\text {add }}$ becomes larger. Table III(b) shows a special set of $\Delta L_{\text {add }}$ given by Doerr [5] without a detailed explanation. Obviously, Table III(b) is a subset of Table III(a). Note that $(1 / 5,3 / 5,1 / 5,0,0)$ in Table III(b) is equivalent to $(0,1 / 5,3 / 5$, $1 / 5,0)$ in Table III(a) for $N=5$, and $(3 / 4,1 / 3,3 / 4,0,1 / 12,0)$ in Table III(b) is equivalent to $(1 / 12,0,3 / 4,1 / 3,3 / 4,0)$ in Table III(a) for $N=6$, etc.

Multiple solutions of $\Delta L_{\text {add }}$ may give additional flexibilities in IAWG design, but more importantly, the general design 
TABLE III

(a) Multiple Sets of Additional Waveguide Lengths $\left[\lambda_{c}\right]$ In the Design of AN $N$-IAWG. (b) Comparisons BETWEEN THE DistRibutions of AdDitional WAVEGUIDE LENGTHS $\left[\lambda_{c}\right]$ IN THE DESIGN OF IAWG

\begin{tabular}{|c|c|c|c|}
\hline$N$ & Our Results & $N$ & Our Results \\
\hline 2 & $1 / 4,0$ & \multirow[t]{6}{*}{7} & $0,1 / 7,3 / 7,6 / 7,3 / 7,1 / 7,0$ \\
\hline \multirow[t]{2}{*}{3} & $1 / 3,0,0$ & & $0,2 / 7,6 / 7,5 / 7,6 / 7,2 / 7,0$ \\
\hline & $2 / 3,0,0$ & & $0,3 / 7,2 / 7,4 / 7,2 / 7,3 / 7,0$ \\
\hline \multirow[t]{3}{*}{4} & $1 / 2,0,0,0$ & & $0,4 / 7,5 / 7,3 / 7,5 / 7,4 / 7,0$ \\
\hline & $1 / 4,1 / 2,1 / 4,0$ & & $0,5 / 7,1 / 7,2 / 7,1 / 7,5 / 7,0$ \\
\hline & $3 / 4,1 / 2,3 / 4,0$ & & $0,6 / 7,4 / 7,1 / 7,4 / 7,6 / 7,0$ \\
\hline \multirow[t]{4}{*}{5} & $0,2 / 5,1 / 5,2 / 5,0$ & \multirow[t]{7}{*}{8} & $0,3 / 4,1 / 2,0,1 / 2,3 / 4,0,0$ \\
\hline & $0,3 / 5,4 / 5,3 / 5,0$ & & $1 / 8,1 / 4,5 / 8,0,5 / 8,1 / 4,1 / 8,0$ \\
\hline & $0,1 / 5,3 / 5,1 / 5,0$ & & $0,0,1 / 4,1 / 2,0,1 / 2,1 / 4,0$ \\
\hline & $0,4 / 5,2 / 5,4 / 5,0$ & & $1 / 8,0,3 / 8,1 / 2,1 / 8,1 / 2,3 / 8,0$ \\
\hline \multirow[t]{6}{*}{6} & $1 / 12,1 / 3,3 / 4,1 / 3,1 / 12,0$ & & $0,1 / 4,0,0,1 / 2,1 / 4,1 / 2,0$ \\
\hline & $11 / 12,0,1 / 4,2 / 3,1 / 4,0$ & & $1 / 8,1 / 4,1 / 8,0,5 / 8,1 / 4,5 / 8,0$ \\
\hline & $3 / 4,2 / 3,3 / 4,0,5 / 12,0$ & & $7 / 8,3 / 4,3 / 8,0,3 / 8,3 / 4,7 / 8,0$ \\
\hline & $1 / 4,1 / 3,1 / 4,0,7 / 12,0$ & & \\
\hline & $1 / 12,0,3 / 4,1 / 3,3 / 4,0$ & & \\
\hline & $11 / 12,2 / 3,1 / 4,2 / 3,11 / 12,0$ & & \\
\hline
\end{tabular}

(a)

\begin{tabular}{|c|c|}
\hline$N$ & Reference [5] \\
\hline 2 & $1 / 4,0$ \\
\hline 3 & $1 / 3,0,0$ \\
\hline 4 & $1 / 2,0,0,0$ \\
\hline 5 & $1 / 5,3 / 5,1 / 5,0,0$ \\
\hline 6 & $3 / 4,1 / 3,3 / 4,0,1 / 12,0$ \\
\hline 7 & $1 / 7,3 / 7,6 / 7,3 / 7,1 / 7,0,0$ \\
\hline 8 & $1 / 4,1 / 2,0,1 / 2,1 / 4,0,0,0$ \\
\hline
\end{tabular}

(b)

rule summarized in the previous section reflects the basic understanding of IAWG and provides a convenient tool for practical device design and optimization.

It should be noted that in the above calculations, $\Omega_{0}$ is always used as the central BZ for convenience, which means that the output ports on the second star coupler have to be arranged in accordance with the locations of BZs, as illustrated in Fig. 4. If $N$ is an odd number, the distribution of waveguides is symmetrical about the center of the output star coupler, while if $N$ is an even number, there is one extra output port on one side in comparison to the other side.

\section{E. Transfer Function of an N-IAWG}

In the previous sections, the general design rule has been derived, and multiple sets of $\Delta L_{\text {add }}$ are calculated. In this section, we will derive the general transfer function of an $N$-IAWG. A 4-IAWG will be used as a numerical example to verify that adding $\Delta L_{\text {add }}$ to the arrayed waveguides does transform an AWG into an IAWG. According to the study in [10], the amplitude distribution of signal in a star coupler is Gaussian. The intensity distribution at the output side of the first star coupler in $N$-IAWG [position 2 in Fig. 4(a)] can be expressed as

$$
I_{0}(\sigma, M, m)=\frac{1}{\sigma \sqrt{2 \pi}} \exp \left[-\frac{\left(m-\frac{M}{2}\right)^{2}}{2 \sigma^{2}}\right]
$$

where $m=1,2, \ldots, M$ is the index of the waveguides, and $M$ is the total number of the waveguides in the array. $\sigma$ is the standard deviation of the distribution. With the Gaussian distribution of the signal at the star coupler as given by (10), the amplitude at one of the output ports of an $N$-IAWG, as shown in Fig. 4(a), can be expressed as

$$
\begin{aligned}
A_{\text {IAWG }}(l, \lambda)=C(l) \cdot \sum_{m=1}^{M} A(m) B(m) \\
\times \exp \left\{j k \cdot \left(\left[L_{0}+(m-1) \cdot \Delta L\right.\right.\right. \\
\left.+\Delta L_{\text {add }}(m-1)\right] \cdot N_{c} \\
+\left[P\left(x_{1}(0),(m-1)\right)\right. \\
\left.\left.\left.+Q\left((m-1), x_{2}(l)\right)\right] \cdot N_{s}\right)\right\}
\end{aligned}
$$

where $l$ is the index of the output ports of the second star coupler, $\lambda$ is the channel wavelength, $L_{0}$ is the length of the shortest waveguide in the waveguide array, and $N_{c}$ is the refractive index of waveguide, while $N_{s}$ is the refractive index of the slab region of the star coupler. $\Delta L$ is the path length difference between adjacent arrayed waveguides of a traditional AWG. $\Delta L_{\text {add }}$ is the additional lengths added to the arrayed waveguides in AWG to obtain IAWG. $A(m)$ is the amplitude distribution at the input side of the arrayed waveguides; $B(\mathrm{~m})$ is a modification factor for the output of the arrayed waveguides transmitting to the output ports of the second star coupler. $C(l)$ is a modification factor to the output at the $l$ th output port of the second star coupler due to Gaussian distribution. $P\left(x_{1}, m\right)$ is the path length from the input port at $x_{1}$ through the first star coupler to the $m$ th arrayed waveguide. $Q\left(m, x_{2}\right)$ is the path 
length from the $m$ th arrayed waveguide through the second star coupler to the output port at $x_{2}$. Here, $m$ is 0 for the shortest waveguide in the array. According to the study in [10]

$$
\begin{aligned}
& P\left(x_{1}, m\right)=R_{0}-\frac{(2 m-1) x_{1} d}{2 R_{0}} \\
& Q\left(m, x_{2}\right)=R_{0}-\frac{(2 m-1) x_{2} d}{2 R_{0}}
\end{aligned}
$$

where $x_{1}$ and $x_{2}$ denote the positions of input port and output port on the corresponding star couplers, as shown in Fig. 4(a). $d$ is the arrayed waveguide separation, and $R_{0}$ is the radius of curvature of star coupler. The distribution factors in (11) can be expressed as

$$
\begin{aligned}
A(m) & =B(m) \\
& =\left[I_{0}\left(\sigma_{0}, M, m\right)\right]^{1 / 2} \\
C(l) & =\left[\left(\sigma_{0} \sqrt{2 \pi}\right) \cdot I_{0}\left(\sigma_{0} / 2, M, l\right)\right]^{1 / 2}
\end{aligned}
$$

where $A(m)=B(m)$ results from the principle of reciprocity. The distribution of $C(l)$ is normalized, and its half width is determined by the Rowland structure of the star coupler. Equation (11) can then be written as

$$
\begin{aligned}
A_{\text {IAWG }}(l, \lambda)=C(l) \cdot \sum_{m=1}^{M} I( & \\
\times \exp \{j k \cdot( & {\left[L_{0}+(m-1) \cdot \Delta L\right.} \\
& \left.+\Delta L_{\text {add }}(m-1)\right] \cdot N_{c} \\
+ & {\left[P\left(x_{1}(0),(m-1)\right)\right.} \\
& \left.\left.\left.+Q\left((m-1), x_{2}(l)\right)\right] \cdot N_{s}\right)\right\}
\end{aligned}
$$

where $I(m)=I_{0}\left(\sigma_{0}, M, m\right)$. Equation (16) is the amplitude transfer function of an $N$-IAWG in which there is only one central input port. For an $N$-IAWG with multiple input ports, its amplitude transfer function can be expressed as

$$
\begin{aligned}
B_{\text {IAWG }}\left(l_{1}, l_{2}, \lambda\right)= & C\left(l_{1}\right) \cdot C\left(l_{2}\right) \cdot \sum_{m=1}^{M} I(m) \\
\times \exp \left\{j k \cdot \left(\left[L_{0}+(m-1) \cdot \Delta L\right.\right.\right. & \left.+\Delta L_{\mathrm{add}}(m-1)\right] \cdot N_{c} \\
& +\left[P\left(x_{1}\left(l_{1}\right),(m-1)\right)\right. \\
& \left.\left.\left.+Q\left((m-1), x_{2}\left(l_{2}\right)\right)\right] \cdot N_{s}\right)\right\}
\end{aligned}
$$

where $C\left(l_{1}\right)$ and $C\left(l_{2}\right)$ are the normalized field distributions at the multiple input ports and output ports of the $N$-IAWG, respectively. $B_{\mathrm{IAWG}}\left(l_{1}, l_{2}, \lambda\right)$ is the transfer function from input waveguide $l_{1}$ to output waveguide $l_{2}$. Clearly, we have

$$
A_{\text {IAWG }}(l, \lambda)=B_{\text {IAWG }}(0, l, \lambda) \text {. }
$$

As an example, Fig. 5 shows the calculated power transfer function for a 4-IAWG. This 4-IAWG is designed to accommodate four channels of wavelengths at 1549.2, 1549.6, 1550.0, and $1550.4 \mathrm{~nm}$, with $\lambda_{0}=1550.0 \mathrm{~nm}$ as the central wavelength. The parameter values needed in (10) are chosen as $M=64, \sigma=\sigma_{0}=0.36 \mathrm{~W} / 2$, where $W=63 D_{0}$ is the width of the output region of the star coupler, with $D_{0}$ as the output waveguide separation. Fig. 5(a) shows the transfer functions from the central input to the four output waveguides $(1,5$, 9, 13), which selects the $\lambda_{0}$ component. This figure clearly demonstrates that the outputs from these four output ports are almost identical, which verifies the characteristic of a 4-IAWG. Similarly, Fig. 5(b)-(d) shows the transfer functions of three other subsets of the output waveguides, as shown in Fig. 4(a), where each subset selects a specific wavelength.

\section{Realization of a $1 \times N$ All-Optical Switch}

\section{A. Transfer Function of a $1 \times N$ All-Optical Switch}

As illustrated in Fig. 2, a $1 \times N$ optical switch can be made using two $N$-IAWGs with an array of phase shifters between them. Based on the transfer function of a single $N$-IAWG given by (16) and (17), the transfer function of a $1 \times N$ optical switch can be easily obtained as the concatenation of the transfer functions of two $N$-IAWGs and an array of phase shifters

$$
\begin{aligned}
A_{\text {4out }}(n, \lambda)=\sum_{l=1}^{N \cdot N} A_{\text {IAWG }}(l, \lambda) \\
\quad \cdot \exp [j \cdot \Delta \theta(l, \lambda)] \cdot B_{\text {IAWG }}(l, n, \lambda)
\end{aligned}
$$

where $A_{\text {IAWG }}(l, \lambda)$ is the transfer function of the input $N$-IAWG, $\exp [j \cdot \Delta \theta(l, \lambda)]$ is the transfer function of the phase shifter array, and $B_{\mathrm{IAWG}}(l, n, \lambda)$ is the transfer function of the output $N$-IAWG.

\section{B. Verification of Switching Functionality and Phase Change Information}

The functionality of an $N$-IAWG has been explained in Section IV-A. To realize the $1 \times N$ switching functionality using the structure shown in Fig. 2, one should be able to switch a signal of wavelength $\lambda_{i}(i=1,2, \ldots, N)$ from the input to any one of the output ports. Because the destination of each $\lambda_{i}$ component is controlled by a dedicated set of phase shifters, there is no contention problem, and the switching of $\lambda_{i}$ will not be influenced by the switch configuration of the other wavelengths.

As mentioned in Section IV, the analysis of the switching functionality is based on reciprocity. If we use star coupler 4 as the input and input the wavelength channel $\lambda_{i}$ into the $n$th waveguide port at interface B in Fig. 2, we should be able to collect its output signal with phase information at the $N$ corresponding waveguide ports at interface A. If the phase of the signal at the $k$ th waveguide port at interface $\mathrm{A}$ is denoted as $\theta_{n}\left(k, \lambda_{i}\right)$, where $k$ is equal to $i, N+i, 2 N+i, \ldots,(N-$ 1) $N+i$, its complex conjugate can be expressed as $\theta_{n}^{*}\left(k, \lambda_{i}\right)$. It is then obvious that if the same wavelength channel is injected 


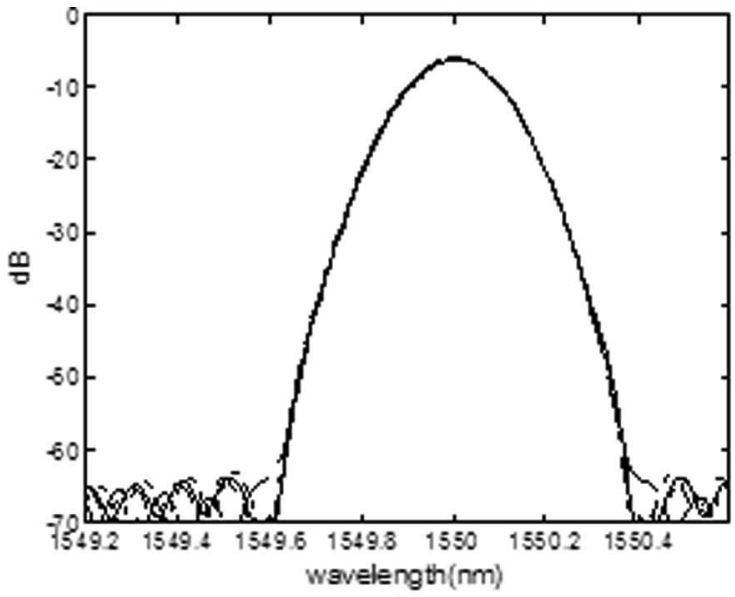

(a)

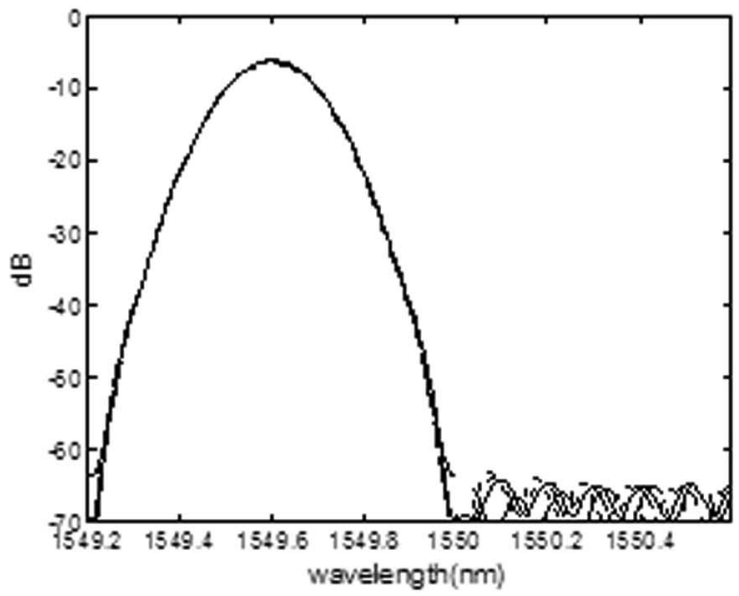

(c)

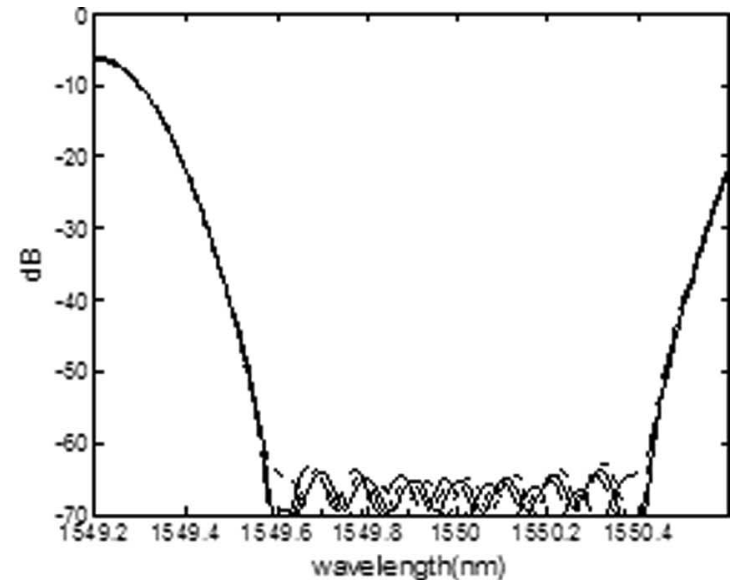

(b)

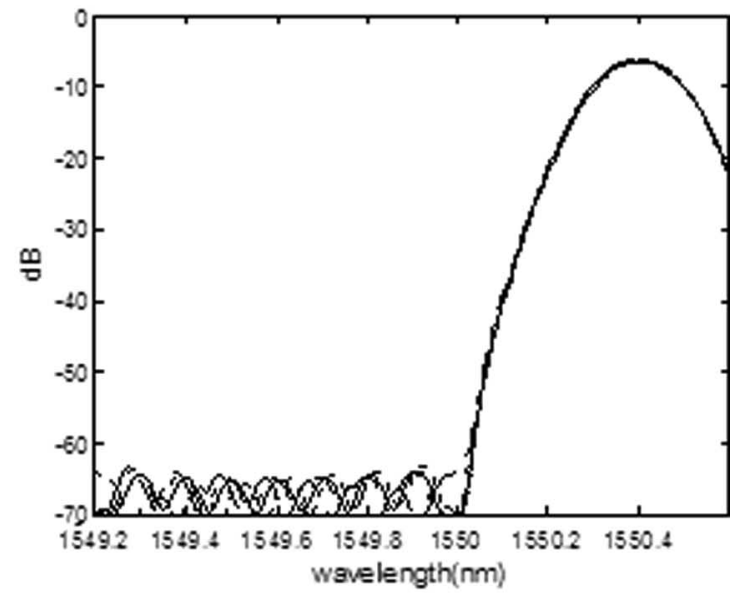

(d)

Fig. 5. Verification of a 4-IAWG. The outputs at its related four output ports for each channel are exactly the same. (a) Central channel with wavelength $\lambda_{0}=1550 \mathrm{~nm}$. (b) Channel of wavelength $1549.2 \mathrm{~nm}$. (c) Channel of wavelength $1549.6 \mathrm{~nm}$. (d) Channel of wavelength $1550.4 \mathrm{~nm}$.

into the central input port of star coupler 1, the signal will be routed to the $n$th output port at star coupler 4 as long as the phase at the $k$ th input port of star coupler 3 at interface A is $\theta_{n}^{*}\left(k, \lambda_{i}\right)$ for all $k=i, N+i, 2 N+i, \ldots,(N-1) N+i$. These conditions can be described by the following equations:

$$
\begin{aligned}
& A_{2 \text { out }}\left(k, \lambda_{i}\right)=\left|A_{\text {2out }}\left(k, \lambda_{i}\right)\right| \exp \left[j \cdot \theta_{0}\left(k, \lambda_{i}\right)\right] \\
& A_{\text {3out }}\left(k, \lambda_{i}\right)=\left|A_{\text {2out }}\left(k, \lambda_{i}\right)\right| \exp \left[j \cdot \theta_{n}^{*}\left(k, \lambda_{i}\right)\right] .
\end{aligned}
$$

As long as (21) is satisfied, the signal of wavelength $\lambda_{i}$ will be routed to the $n$th output port of star coupler 4 at interface B. From (20) and (21), the phase adjustment required in the $k$ th phase shifter can be obtained as

$$
\Delta \theta\left(k, \lambda_{i}\right)=\theta_{n}^{*}\left(k, \lambda_{i}\right)-\theta_{0}\left(k, \lambda_{i}\right)
$$

$\theta_{0}\left(k, \lambda_{i}\right)$ in (20) and (22) is the reference phase of channel $\lambda_{i}$ at the $k$ th output port of star coupler 2 . To route any wavelength channel from the input port to any output port, every phase shifter needs an independent control signal to obtain the required phase adjustment. Since there are $N^{2}$ phase shifters, as shown in Fig. 2, there should be $N^{2}$ degrees of freedom in this $1 \times N$ structure.
Based on the above discussion, the results of the $1 \times 4$ switch are shown in Fig. 6, which were calculated from the transfer functions given by (19). This $1 \times 4$ switch was designed to accommodate four wavelength channels at 1549.2, 1549.6, 1550.0 , and $1550.4 \mathrm{~nm}$. Fig. 6(a)-(d) shows that the 1550.0-nm channel, the 1549.6-nm channel, the 1550.4-nm channel, and the 1549.2-nm channel are switched to output ports 1, 2, 3, and 4 , respectively. For comparison, these four graphs are combined as shown in Fig. 7. It indicates that the worst-case crosstalk between channels is about $-32 \mathrm{~dB}$. This crosstalk level may be further reduced by apodizing and other AWG optimization techniques [10], which are outside the scope of this paper.

The phase adjustments required in the phase shifters in this example are presented in Table IV. To obtain Table IV, we have assumed that the equal length waveguides are connected to the phase shifters for convenience.

The phase shifters can be sections of waveguides made from semiconductor materials. The refractive index in these waveguides can be changed due to band filling, band shrinkage, and free-carrier absorption effects [11]. By changing the refractive indexes of the waveguides, the phases of the optical signals passing through them can be changed. In waveguides made of semiconductors, the refractive index is a function of the carrier concentration. For example, the carrier-induced 
(a)

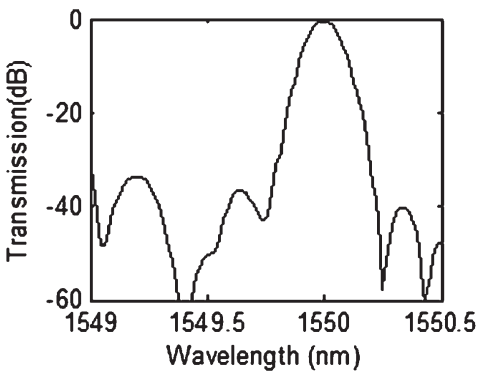

(c)

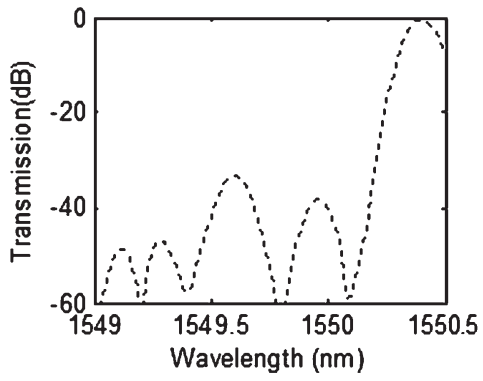

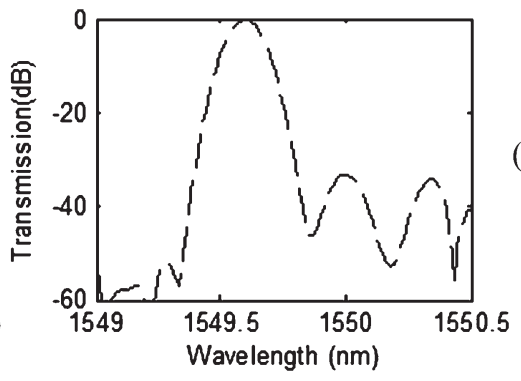

(b)

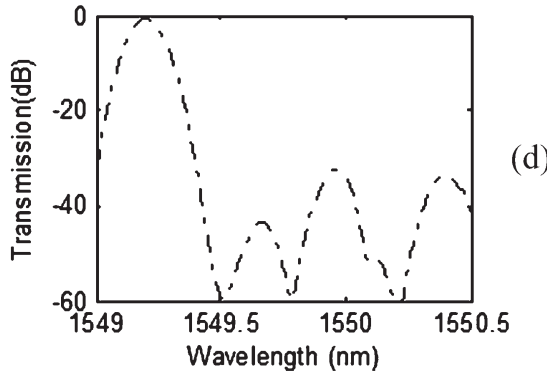

Fig. 6. Transfer functions of a four-wavelength $1 \times 4$ switch designed for operating wavelengths of $1549.2,1549.6,1550.0$, and $1550.4 \mathrm{~nm}$. (a) The $1550.0-\mathrm{nm}$ channel is switched to output port 1; (b) the 1549.6-nm channel is switched to output 2; (c) the 1550.4-nm channel is switched to output 3; and (d) the 1549.2-nm channel is switched to output 4 .

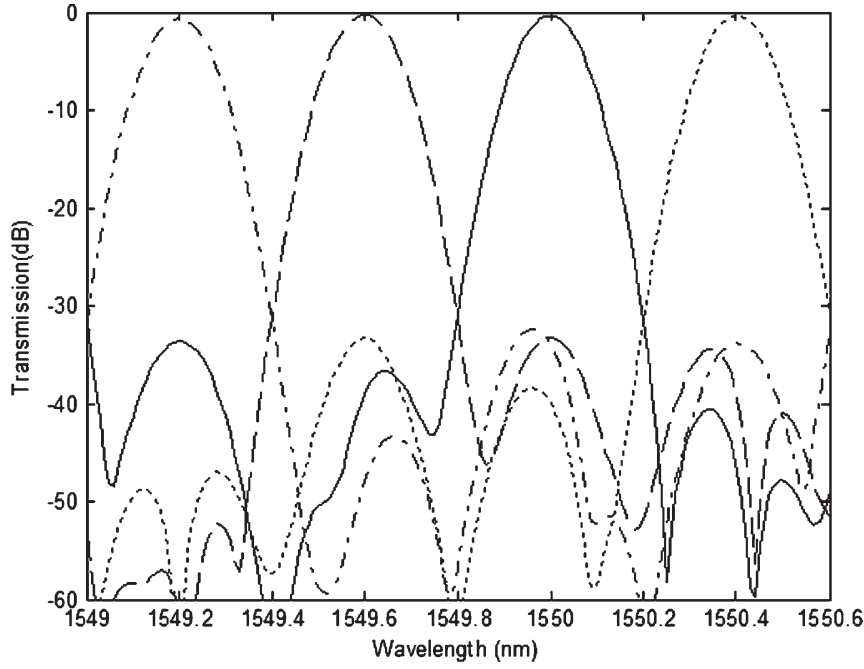

Fig. 7. Transfer function of a $1 \times 4$ switch for four wavelength channels with wavelengths at $1549.2,1549.6,1550.0$, and $1550.4 \mathrm{~nm}$. The crosstalk between channels is around $-32 \mathrm{~dB}$ or less.

refractive index change of $\mathrm{GaN}$ is $\sim 0.03$ at a wavelength of $1550 \mathrm{~nm}$ with a carrier concentration change of $7 \times 10^{18}$ [4], [12]. The carrier-induced refractive index change in InP is $\sim 0.025$ at a wavelength of $1550 \mathrm{~nm}$ for a carrier concentration change of $3 \times 10^{18}$ [11].

The relationship between phase changes and the related refractive index changes can be obtained as

$$
\Delta n=\frac{\lambda}{2 \pi\left(L_{p}\right)} \cdot \Delta \theta
$$

where $L_{P}$ is the length of the phase shifters. From (23), we calculated that the maximum refractive index change needed in a phase shifter of $1-\mathrm{mm}$ length is $1.4 \times 10^{-3}$ to switch a signal in the 1550-nm wavelength region by introducing a phase shift
$\Delta \theta=\pi$. This value of index change is readily achievable with several semiconductor materials [4], [11], [12].

\section{Simplified $1 \times N$ SWITCH BASED ON REFLECTIVE STRUCTURE}

Almost all AWG-based optical switches proposed so far [2], [3], [5], including the $1 \times N$ WDM switch shown in Fig. 2, require two $N$-IAWGs. The two $N$-IAWGs perform largely redundant operations based on the symmetry of the structure. Any structural mismatch between the two $N$-IAWGs may cause asymmetry in their optical transfer functions, thus introducing extra phase mismatch and signal energy loss. Furthermore, the requirement of two $N$-IAWGs makes the physical size of AWG-based optical switches relatively large.

A good understanding of the operating principle, as described in the previous sections, enables us to modify the structural design and optimize the performance of the AWGbased optical switches. Since the functionality of the two $N$ IAWGs in the $1 \times N$ switch structure is almost identical, it should be possible to combine them into one. We propose a simplified $1 \times N$ switch structure, as shown in Fig. 8, where only one $N$-IAWG is required, and a total reflection mirror is used at the end of each phase shifter. This total reflection can be easily obtained by cleaving and high reflection coating at the end of the waveguides. This simplification greatly reduces the complexity of the structure and cuts the size of the AWG-based $1 \times N$ switch by half.

To simplify the structure from that shown in Fig. 2 to that in Fig. 8, the input and output ports must be rearranged. All but one waveguide port at star coupler 1 in Fig. 8 are output ports, and the central port is used as the input port. Therefore, there are only $N-1$ output ports in this design. Although star coupler 1 has $N$ input ports, it only makes a $1 \times(N-1)$ optical switch. By using the $N$-IAWG in a bidirectional manner for both input and output, it guarantees the same transfer characteristics for 
TABLE IV

Phase Changes $(\Delta \theta)$ Needed in Phase Shifters for Each Output Port Selection in a $1 \times 4$ All-Optical Switch $(\lambda=1550 \mathrm{~nm})$

\begin{tabular}{|l|r|r|r|r|}
\hline $\begin{array}{l}\text { Output } \\
\text { Choice }\end{array}$ & Phase shifter 1 & Phase shifter 2 & Phase shifter 3 & Phase shifter 4 \\
\hline Port 1 & 1.659 & -3.128 & 1.510 & 3.007 \\
\hline Port 2 & 0.8362 & -0.8091 & -2.454 & 2.184 \\
\hline Port 3 & -3.128 & 1.510 & 3.007 & 1.362 \\
\hline Port 4 & -0.8091 & 0.6874 & 2.184 & -2.602 \\
\hline
\end{tabular}

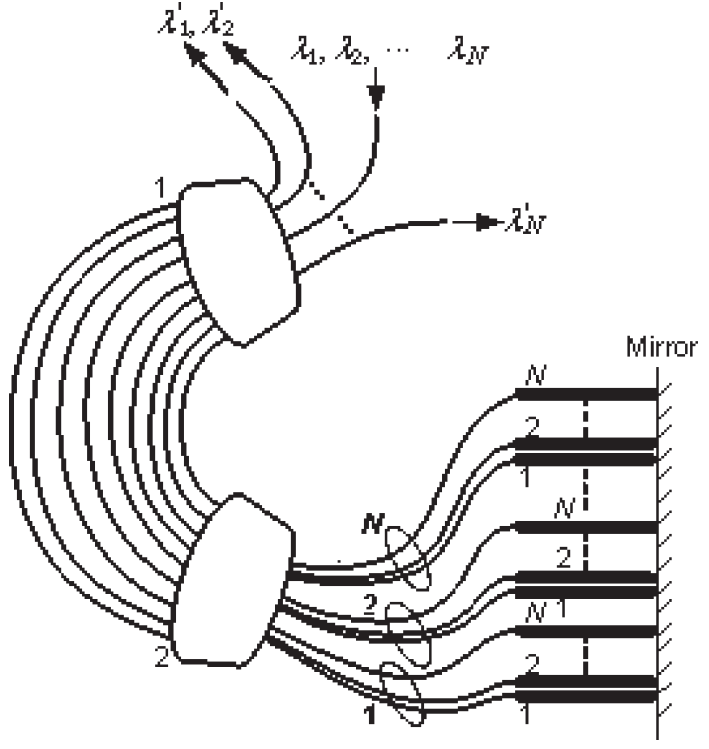

Fig. 8. Mirror scheme of a $1 \times N$ all-optical switch.

the signal propagating before and after the phase shifter array, and the possibility of transfer function mismatch between them is avoided. In addition, the phase shifters are more effectively utilized in this simplified configuration because every phase shifter is used twice due to total reflection at the end surface. This doubles the tuning efficiency compared to the single-pass phase shifter. Since the phase shifters are no longer used to connect between two $N$-IAWGs, they can be much shorter and more flexible in the layout. The reduced device size and more efficient phase tuning will help to integrate the building block into more sophisticated optical devices such as the $N \times N$ alloptical switch shown in Fig. 1. We also calculated the transfer function of this simplified $1 \times N$ optical switch using steps outlined in Section V. Fig. 9 shows examples of switching the input signal to one of the three output ports, respectively, in the structure with $N=4$. Except for the one less output channel, this simplified structure shows the same switching functionality and a similar transfer function as the one requiring two $N$-IAWGs.

\section{CONCLUSION}

In this paper, we proposed a fully functional nonblocking $N \times N$ WDM switch that uses $1 \times N$ optical switches as building blocks. Since a $1 \times N$ optical switch can be constructed based on $N$-IAWGs and a phase shifter array, we developed and presented the general rule for the design of an $N$-IAWG. Compared with conventional AWG, an $N$-IAWG requires unique differential path length design in the arrayed

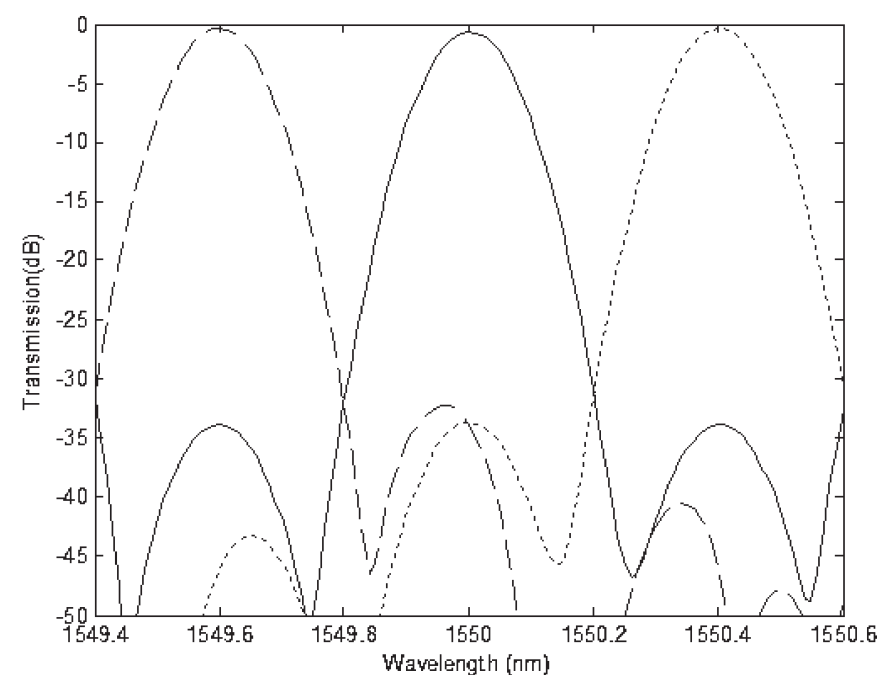

Fig. 9. Transfer function of the mirror scheme of a $1 \times(N-1)$ all-optical switch for $N=4$.

waveguides between the two star couplers. The general design rule reveals that there are multiple solutions to the differential path lengths, which automatically includes the special solution given by Doerr [5]. The steps of designing $1 \times N$ WDM switches were outlined. The related transfer functions and switching functionalities were discussed in detail.

Finally, a simplified $1 \times(N-1)$ WDM switch that only requires a single $N$-IAWG was presented, where the total reflection is implemented at the end of each phase shifter, and the $N$-IAWG is used bidirectionally. This simplified structure significantly reduces the device size and relaxes the design tolerance.

\section{ACKNOWLEDGMENT}

The authors would like to thank Dr. C. Allen for his useful comments.

\section{REFERENCES}

[1] G. Keiser, Optical Fiber Communications, 3rd ed. Boston, MA: McGraw-Hill, 2000.

[2] C. R. Dorr, C. H. Joyner, L. W. Stulz, and R. Monnard, "Wavelengthdivision multiplexing cross connect in InP," IEEE Photon. Technol. Lett., vol. 10, no. 1, pp. 117-119, Jan. 1998.

[3] C. G. P. Herben, D. H. P. Maat, X. J. M. Leijtens, M. R. Leys, Y. S. Oei, and M. K. Smit, "Polarization independent dilated WDM cross-connect on InP," IEEE Photon. Technol. Lett., vol. 11, no. 12, pp. 1599-1601, Dec. 1999.

[4] R. Hui et al., "III-nitride-based planar lightwave circuits for long wavelength optical communications," IEEE J. Quantum Electron., vol. 41, no. 1, pp. 100-110, Jan. 2005.

[5] C. R. Doerr, "Proposed WDM cross connect using a planar arrangement of waveguide grating routers and phase shifters," IEEE Photon. Technol. Lett., vol. 10, no. 4, pp. 528-530, Apr. 1998. 
[6] I. Kaminow and T. Li, Optical Fiber Telecommunications IV A Components. San Diego, CA: Academic, 2002, ch. 9.

[7] H. Takahashi, K. Oda, H. Toba, and Y. Inoue, "Transmission characteristics of arrayed waveguide $N \times N$ wavelength multiplexer," J. Lightw. Technol., vol. 13, no. 3, pp. 447-455, Mar. 1995.

[8] M. Frank et al., BeamPROP AWG Utility Version 2.1, Utility Manual. Ossinning, NY: Rsoft, Inc., 2003.

[9] C. Dragone, "Optimum design of a planar array of tapered waveguides," J. Opt. Soc. Amer. A, Opt. Image Sci., vol. 7, no. 11, pp. 2081-2091, Nov. 1990.

[10] K. Okamoto, Fundamentals of Optical Waveguides. New York: Academic, 2000.

[11] B. R. Bennett, R. A. Soref, and J. A. Del Alamo, "Carrier-induced change in refractive index of InP, GaAs, and InGaAsP," IEEE J. Quantum Electron., vol. 26, no. 1, pp. 113-122, Jan. 1990.

[12] R. Hui et al., "GaN-based waveguide devices for long-wavelength optical communications," Appl. Phys. Lett., vol. 82, no. 9, pp. 1326-1328, Mar. 2003.

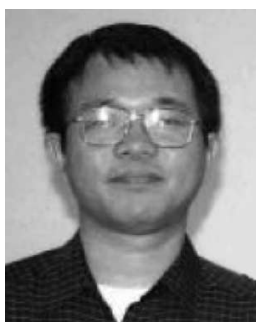

Yueting Wan received the B.S. degree in physics from the University of Science and Technology of China, Anhui, China, in 1987, the M.Eng degree in telecommunications from the Asian Institute of Technology, Bangkok, Thailand, in 1997, the M.S. degree in physics from the University of Memphis, Memphis, TN, in 1999, and the Ph.D. degree in electrical engineering from the University of Kansas, Lawrence, in 2006.

From 1987 to 1996, he taught college physics with Jiangsu University of Science and Technology, Jiangsu, China. He is currently with Harbor Branch Oceanographic Institution, Fort Pierce, FL. His research interests include all-optical switch design and test, novel semiconductor materials in the applications of fiber-optic telecommunications, and LIDAR systems.

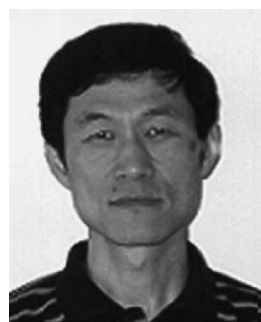

Rongqing Hui received the B.S. degree in microwave communications and the M.S. degree in lightwave technology from Beijing University of Posts and Telecommunications, Beijing, China, in 1981 and 1988, respectively, and the Ph.D. degree in electronics engineering from Politecnico di Torino, Torino, Italy, in 1993.

From 1989 to 1990, he held a research fellowship with the Fundazione Ugo Bordoni, Rome, Italy, working on nonlinear effects and optical injection locking of semiconductor laser devices. From 1990 to 1993, he was with the Department of Electronics, Politecnico di Torino, where he worked on optical communication systems and single-frequency semiconductor lasers. During this period, he also worked as a Research Engineer with the Telecommunications Research Center (CSELT), Torino, Italy. From 1993 to 1994, he was a Postdoctoral Research Fellow with the University of Ottawa, Ottawa, ON, Canada, where he was working on optical networks architecture. He joined Bell-Northern Research (now part of Nortel Networks), Ottawa, in 1994, as a member of scientific staff, where he worked in the research and development of high-speed optical transport networks. Since 1997, he has been with the Department of Electrical Engineering and Computer Science, University of Kansas, Lawrence, where he is currently a Professor. As an author or coauthor, he has published more than 80 referred technical papers in the area of optical communications and photonics devices. He is the holder of 11 patents.

Dr. Hui is currently serving as a Program Director of Photonics and Optoelectronics within the ECCS Division at the National Science Foundation, Arlington VA. He also serves as an Associate Editor for the IEEE JOURNAL OF QUANTUM ELECTRONICS and the IEEE TRANSACTIONS ON COMMUNICATIONS. 